\title{
Tuning PEDOT:Tos thermoelectric properties through nanoparticle inclusion
}

¿DEGLI STUDI

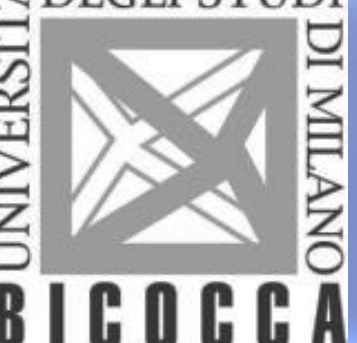

Daniela Galliani

University of Milano-Bicocca

Material Science Department 


\section{Outline}

1. Introduction

- Conjugated Polymers as Thermoelectric Materials

- Conjugated Polymer Nanocomposite

- Energy Filtering Effect

2. Experimental Work

- $\mathrm{Mn}_{3} \mathrm{O}_{4}$ Nanoparticles

- Nanoparticle Functionalization

- Hybrid Film Making

3. Results

- Thermoelectrical Characterization

- Nanoparticle Influence on Polymer Morphology

- Humidity Effect

4. Conclusion and Further Developments 


\section{Conjugated Polymers as}

Introduction

\section{Thermoelectric Materials}

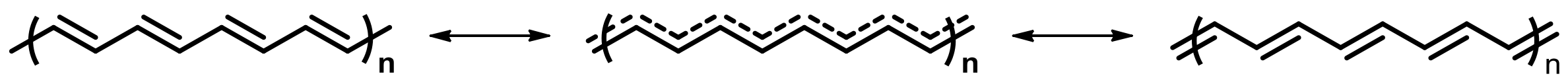

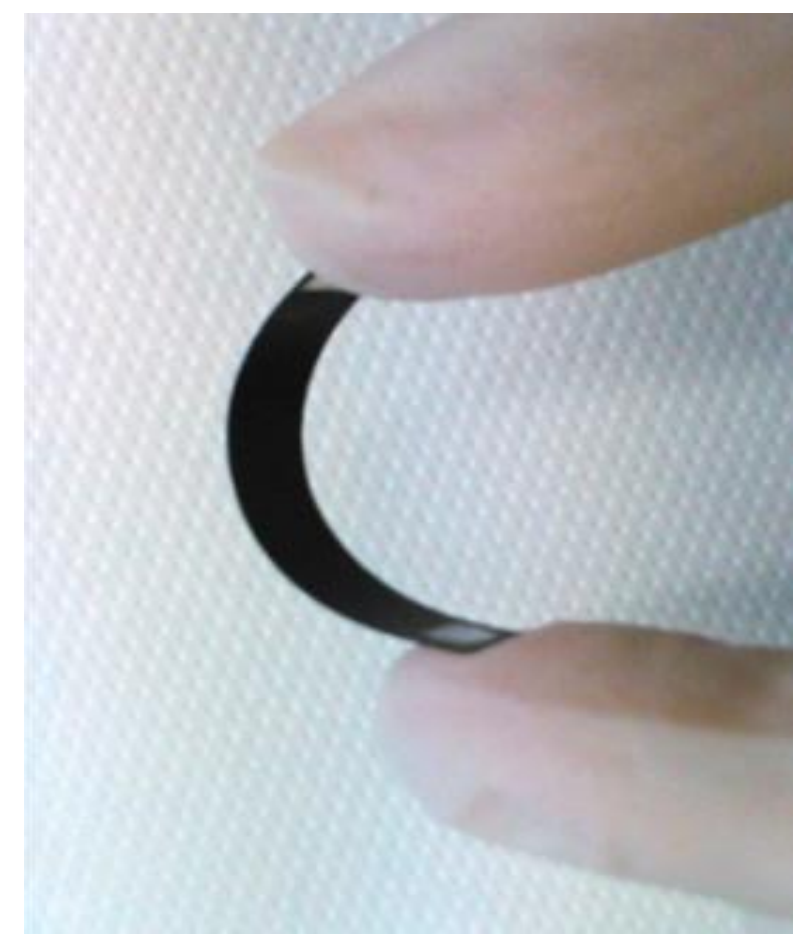

- Low thermal conductivity

- Medium-high electrical conductivity

- Tunable electronic properties

- Easy processing

- Safety

- Environmental stability

- Low cost

- Adaptability to flexible substrate 


\section{Conjugated Polymers as \\ Thermoelectric Materials}

Introduction

\section{Poly(3.4-ethylendioxythiophene) PEDOT}

\section{Low TE efficiency}

a<smiles>CC(C)(C)c1sc(-c2sc(-c3sc(-c4sc(-c5sc(-c6sc(-c7sc(C(C)(C)C)c8c7OCCO8)c7c6OCCO7)c6c5OCCO6)c5c4OCCO5)c4c3OCCO4)c3c2OCCO3)c2c1OCCO2</smiles>

b

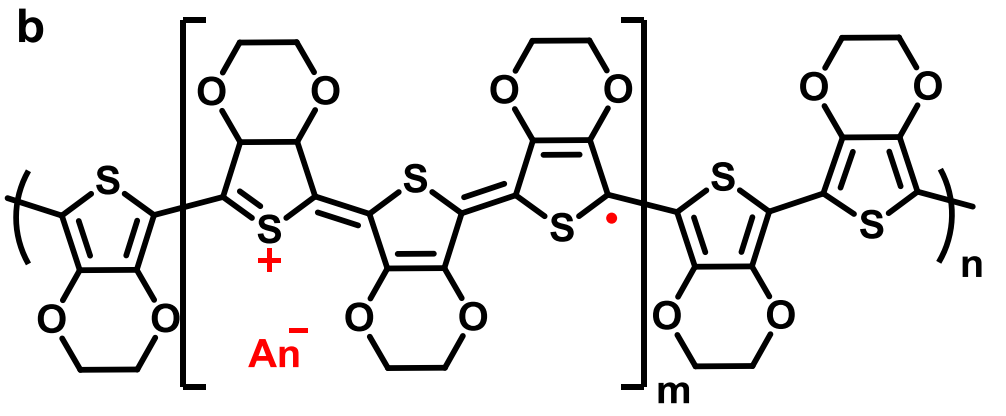

Nanostructuration

$$
\mathrm{An}^{-}=\mathrm{Tos}^{-}, \mathrm{Cl}^{-}, \mathrm{PSS}^{-}, \mathrm{ecc}
$$




\section{Conjugated Polymer}

Introduction

\section{Nanocomposite}

\begin{tabular}{ccccc}
\hline Materials & $\mathbf{S}(\boldsymbol{\mu V} / \mathbf{K})$ & $\mathbf{P F}\left(\boldsymbol{\mu W} / \mathbf{m ~ K}^{2}\right)$ & $\boldsymbol{Z T}$ & Year \\
\hline PEDOT:PSS/SWCNT & 30 & 25 & 0.02 & 2013 \\
PEDOT:PSS/MWCNT & 70 & 500 & - & 2010 \\
PEDOT:PSS/Bi 2 Te & 60 & 130 & 0.1 & 2010 \\
PEDOT:PSS/Te & 163 & 70.9 & 0.1 & 2013 \\
PEDOT:PSS/Au NPs & 26.5 & 51.2 & $\sim 0.1$ & 2014 \\
PEDOT:PSS/Au nanorods & 12 & 30 & - & 2014 \\
PEDOT:PSS/Ge & $\sim 50$ & 165 & 0.1 & 2014 \\
\hline
\end{tabular}

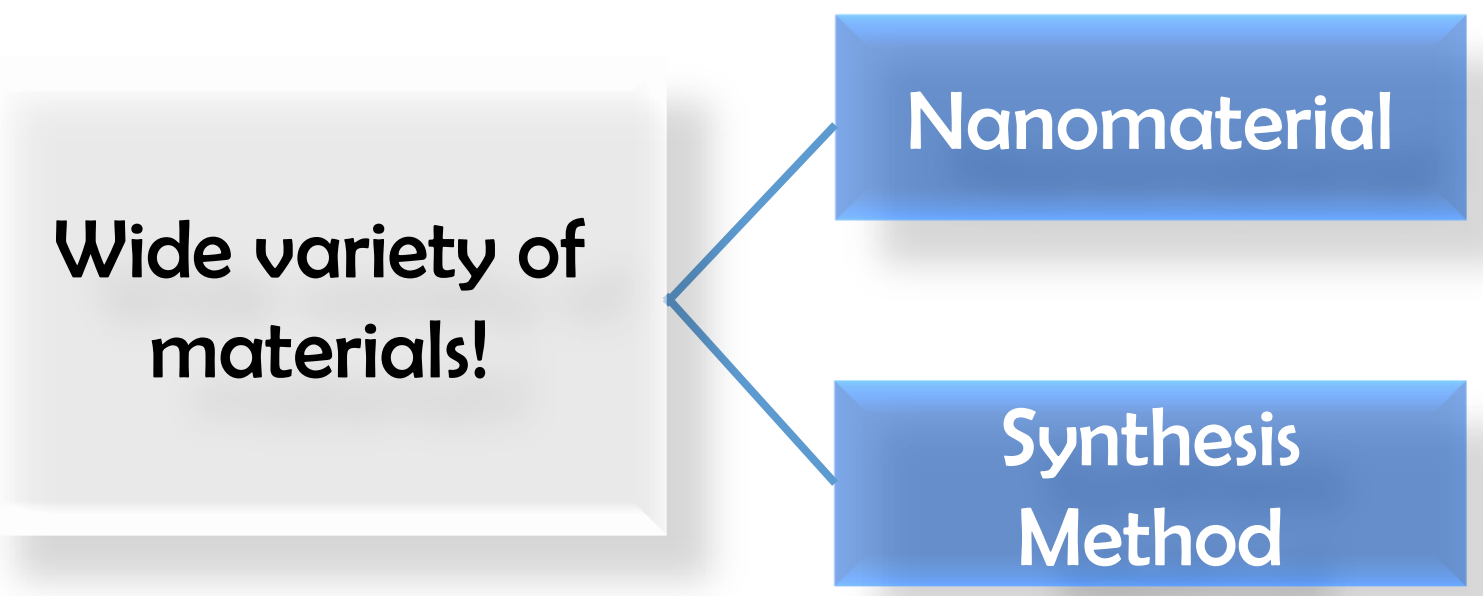

CHOOSING CRITERIA 
Introduction

\section{Energy Filtering Effect}

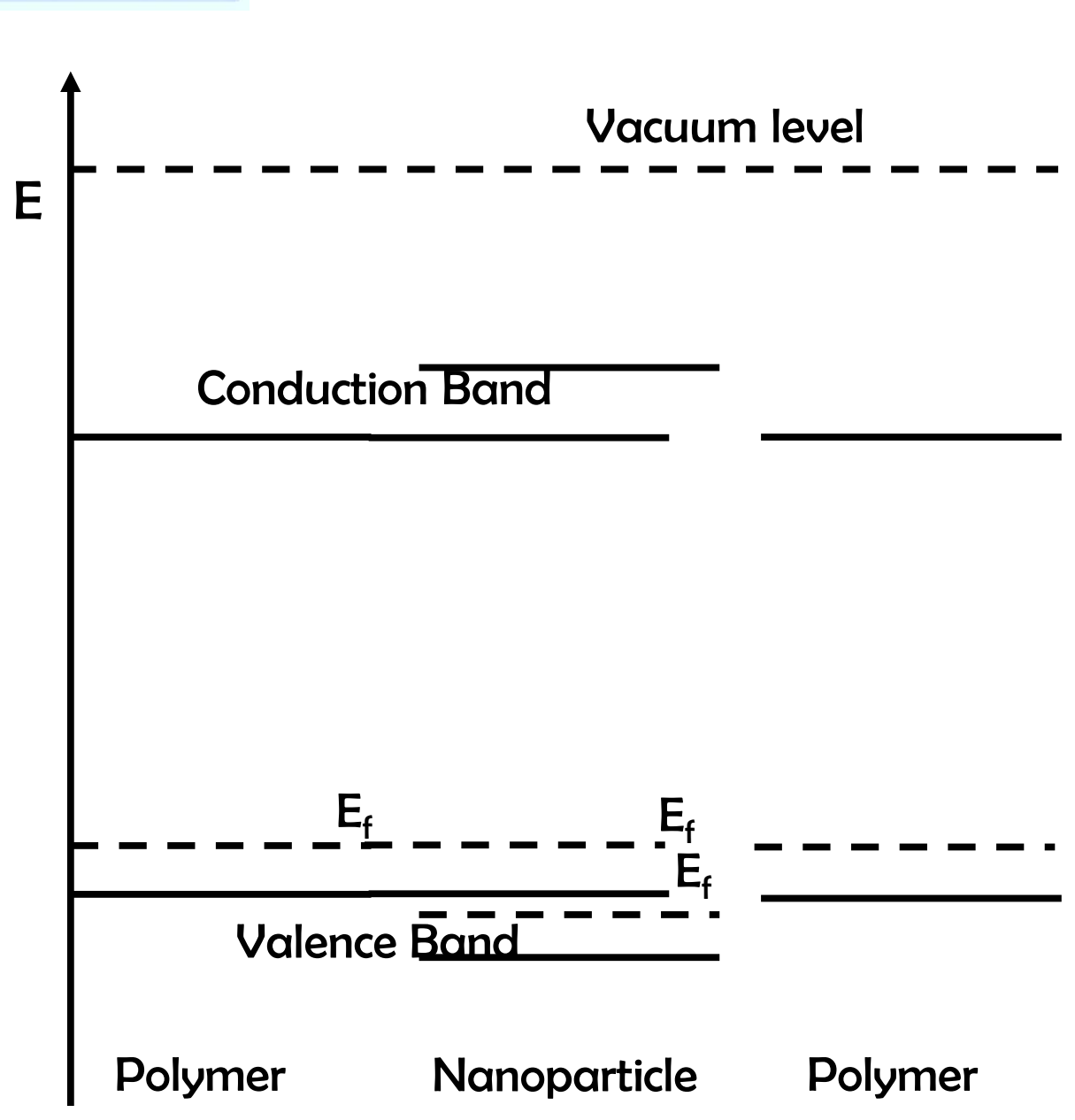

Amorphous zone 
Introduction

\section{Energy Filtering Effect}

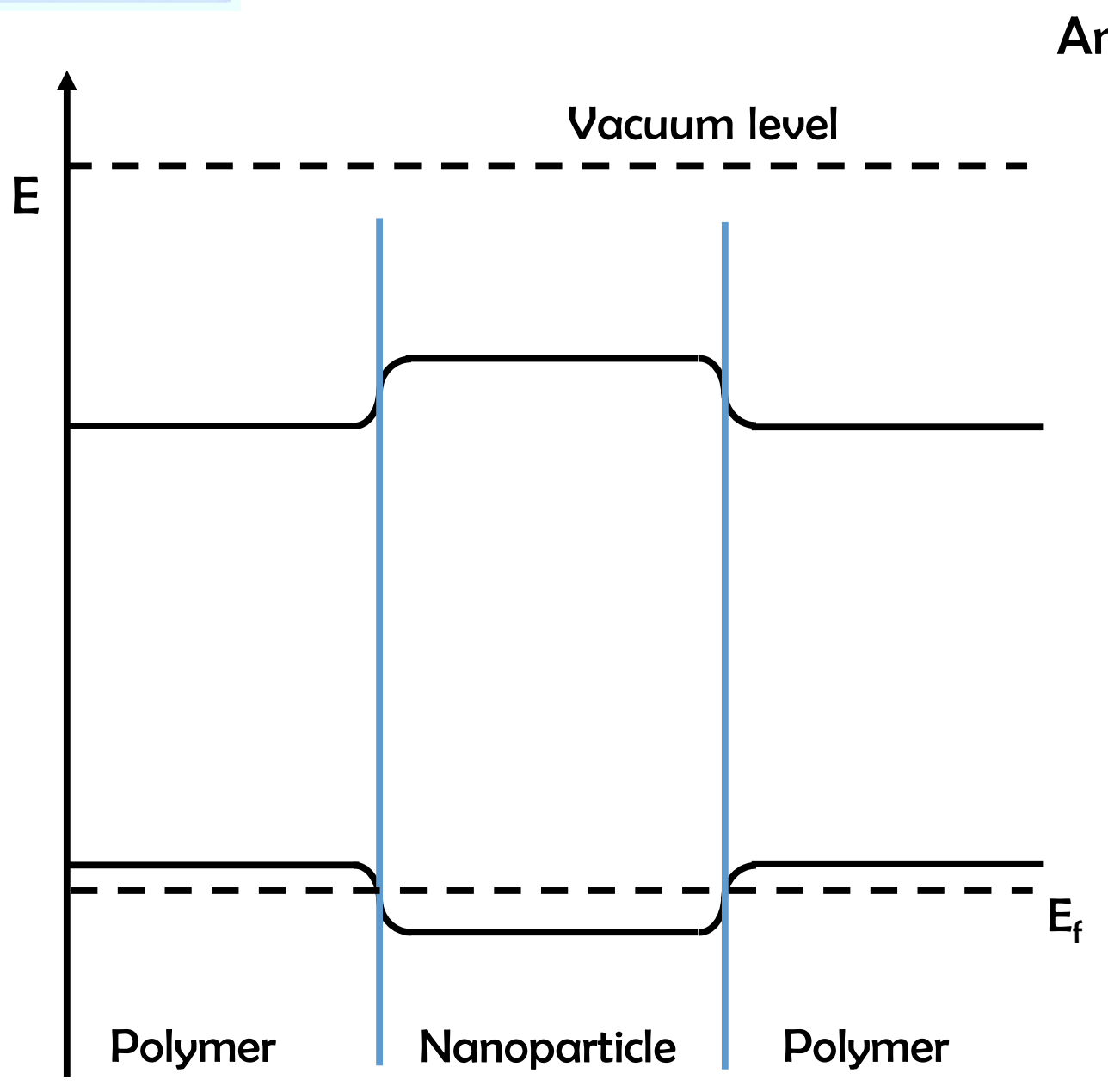

Amorphous zone

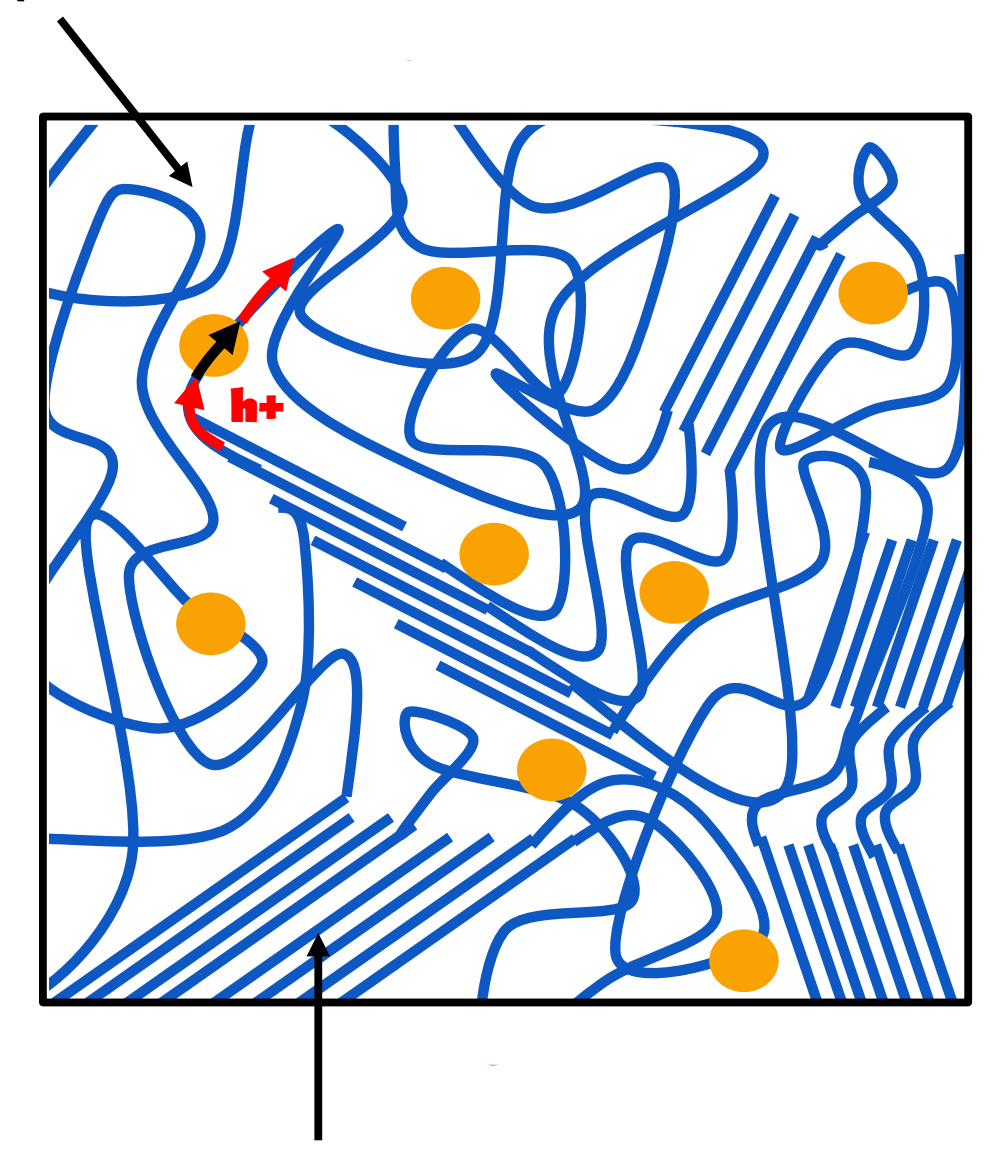

Crystalline domains 
Introduction

\section{Energy Filtering Effect}

University of Milano-Bicocca

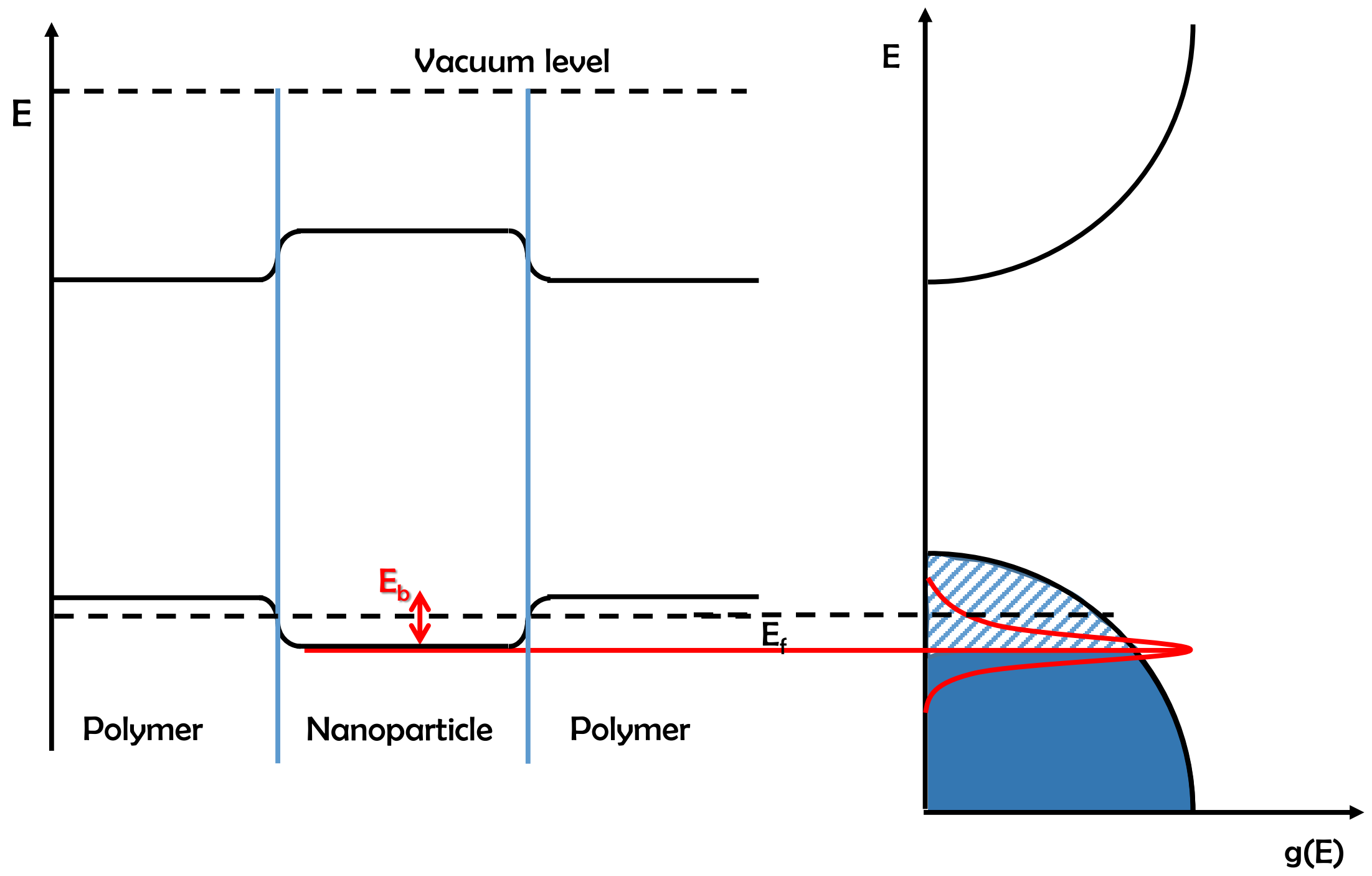




\section{Energy Filtering Effect}

\section{CHOOSING CRITERIA}

- Intimate contacts between CP and NPs

- Similar work functions of the CP and the NPs

- Interfacial barrier height below $100 \mathrm{meV}$
- Chemical interaction between CP and NPs

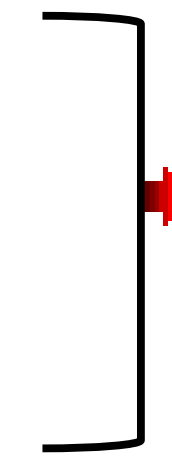

Choice of CP and NP material 


\section{Outline}

1. Introduction

- Conjugated Polymers as Thermoelectric Materials

- Conjugated Polymer Nanocomposite

- Energy Filtering Effect

2. Experimental Work

- $\mathrm{Mn}_{3} \mathrm{O}_{4}$ Nanoparticles

- Nanoparticle Functionalization

- Hybrid Film Making

3. Results

- Thermoelectrical Characterization

- Nanoparticle Influence on Polymer Morphology

- Humidity Effect

4. Conclusion and Further Developments 
$\mathrm{Mn}_{3} \mathrm{O}_{4}$

p-type material

work function: $4,4 \mathrm{eV}$

Thanks to Dr. Simone Battiston IENI-CNR, Padova

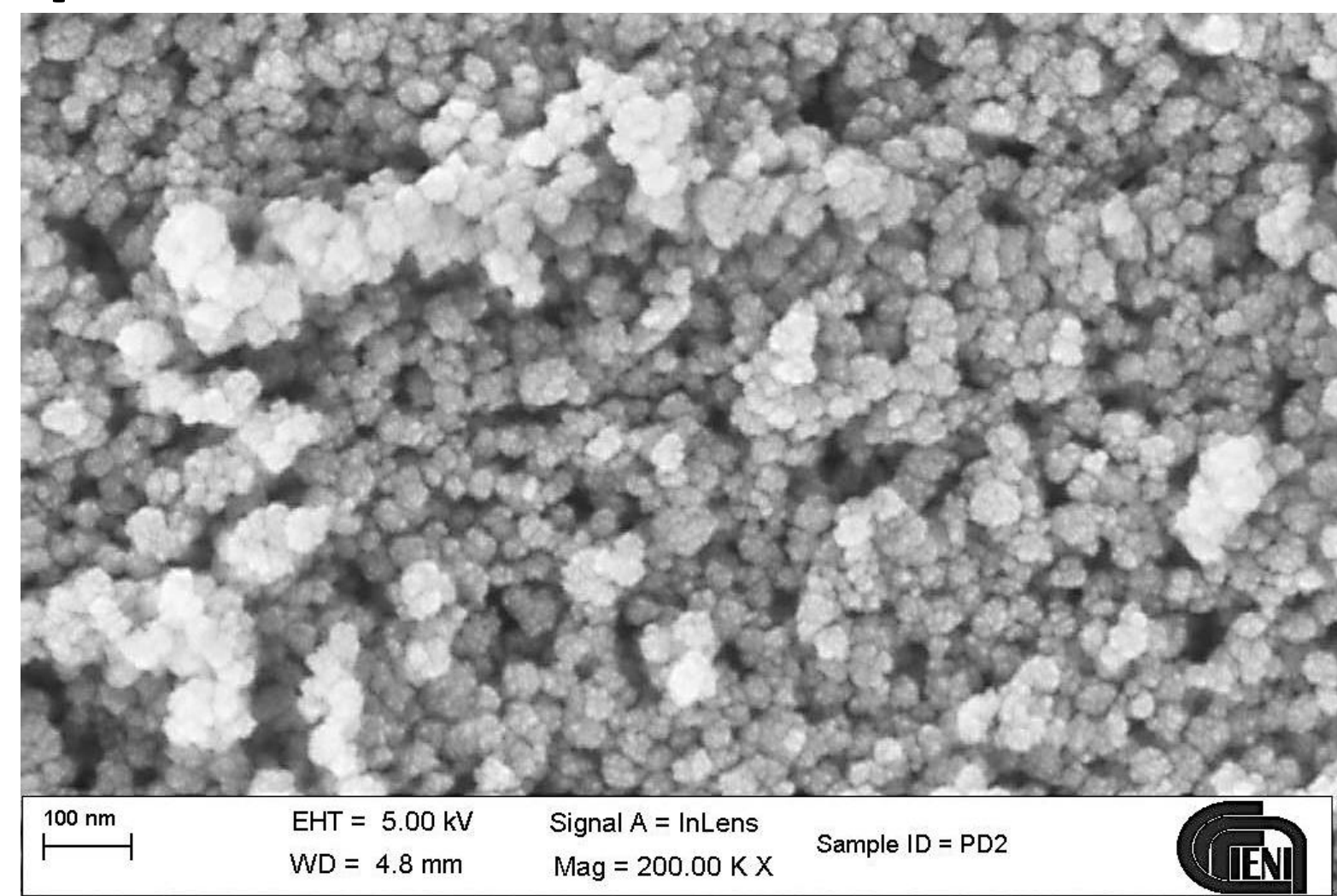

Starting salt Size control agent

Reagent

$\mathrm{T}\left({ }^{\circ} \mathrm{C}\right)$

Size SEM determined $(\mathrm{nm})$

$\mathrm{MnCl}_{2} 4 \mathrm{H}_{2} \mathrm{O}$

Ethanolamine

$\mathrm{H}_{2} \mathrm{O}$

25

$25 \pm 6$ 


\section{Functionalization}

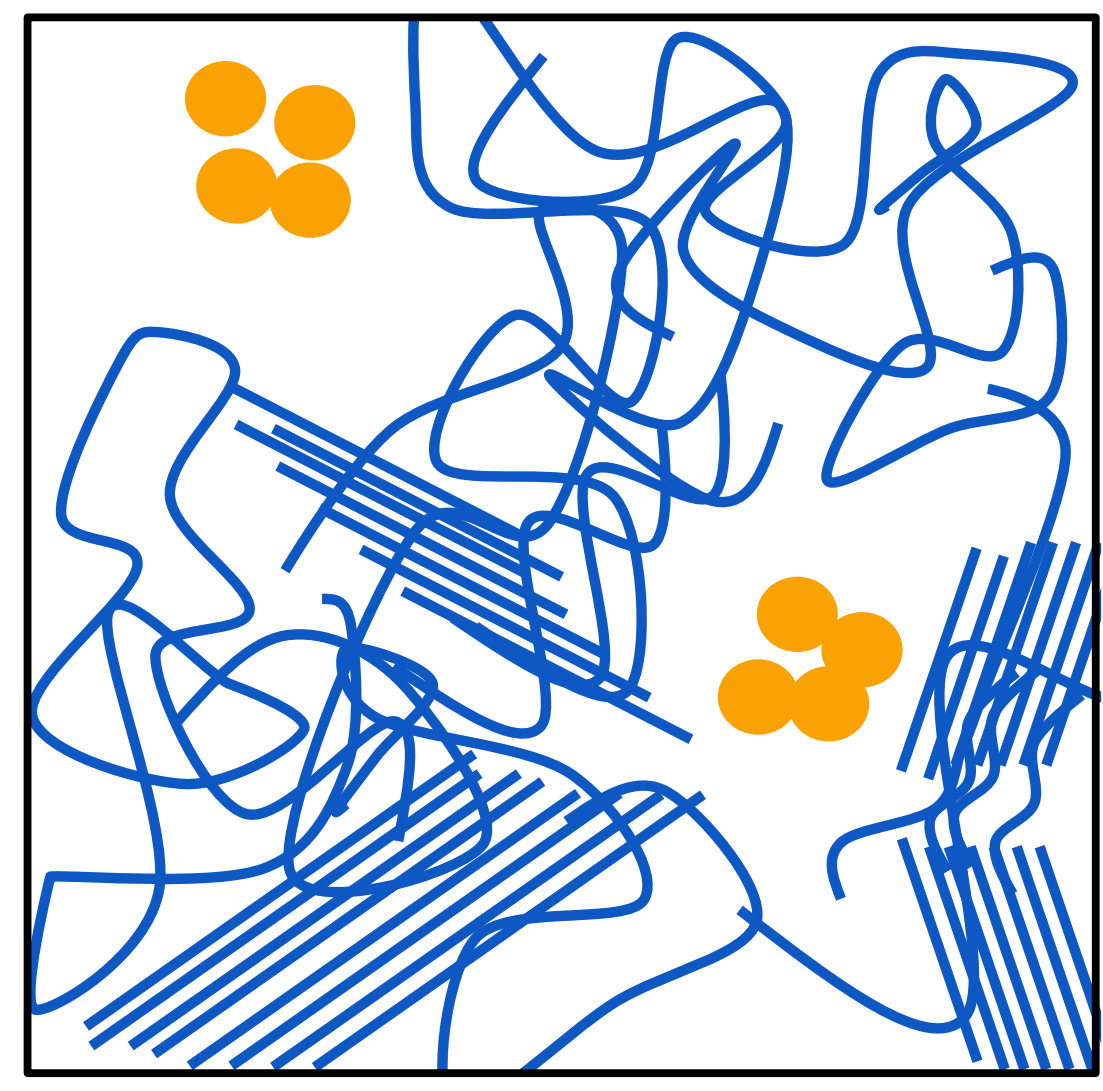

\Phase separation

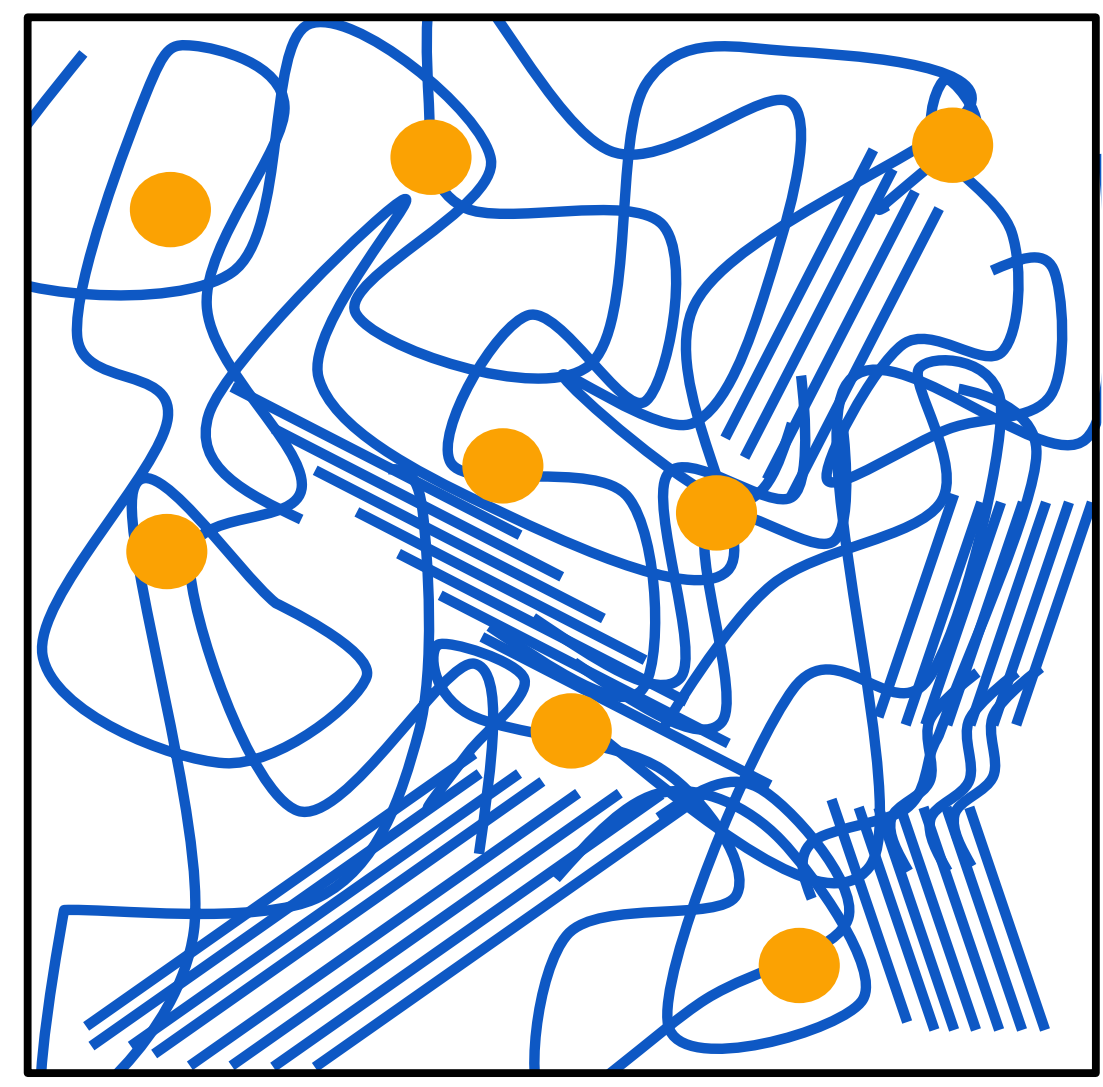

Homogeneous dispersion 


\section{Nanoparticle}

Work

\section{Functionalization}

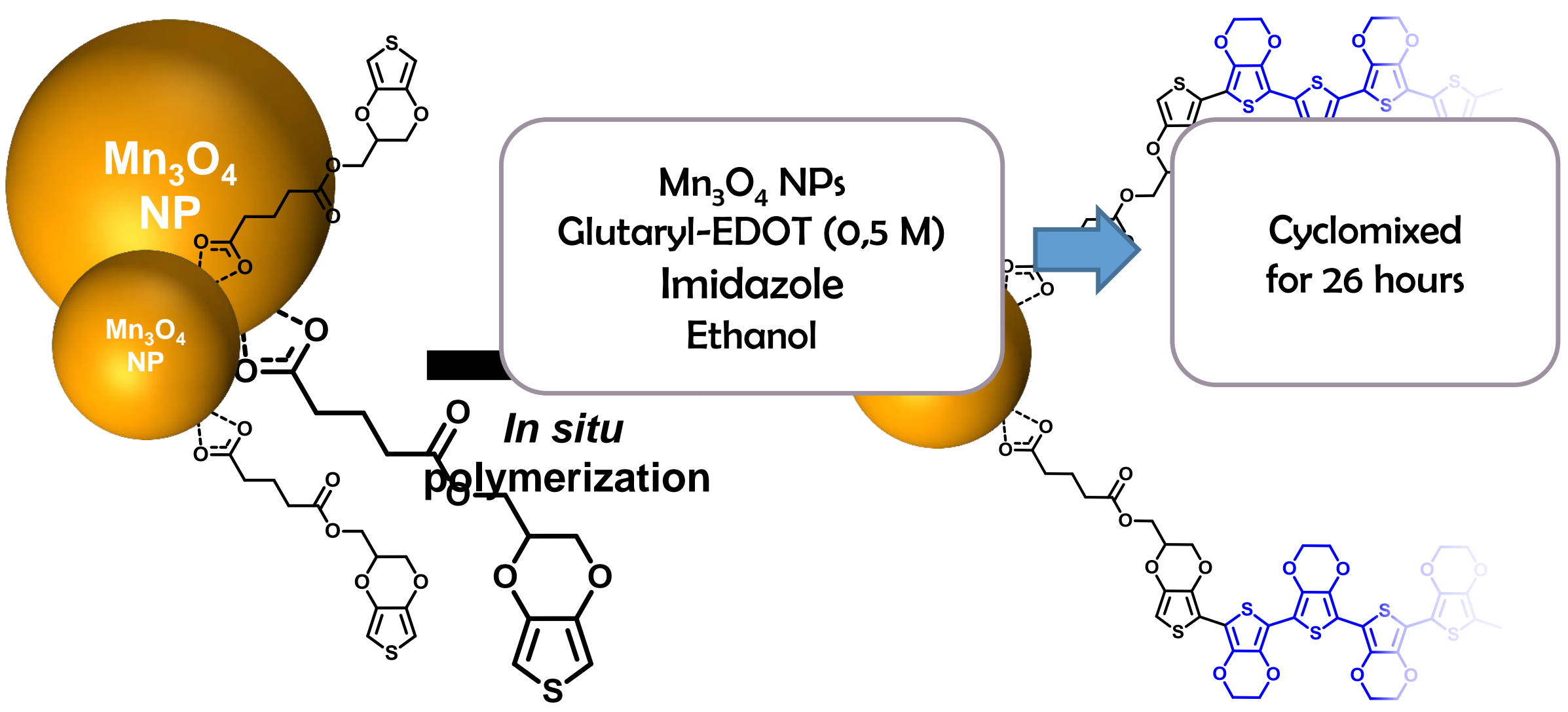

Glutaryl-EDOT 
Work

\section{Blade Coating}

$\mathrm{Mn}_{3} \mathrm{O}_{4} \mathrm{NPs}$ decorated with glutaryl-EDOT

\section{EDOT}

Polumerization in situ

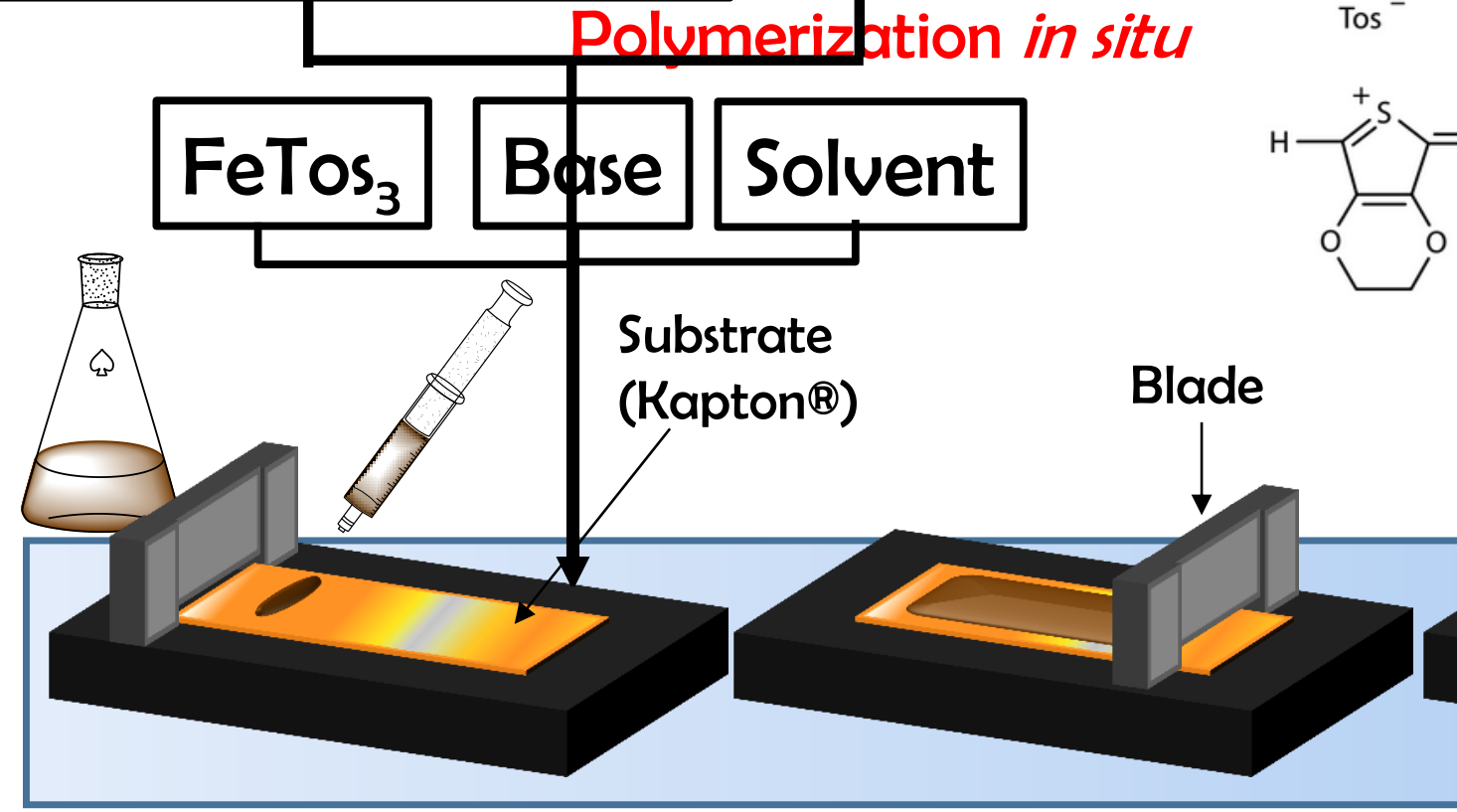

1) Solution deposition
2) Solution spreading
3) Film drying 


\section{Outline}

\section{Introduction}

- Conjugated Polymers as Thermoelectric Materials

- Conjugated Polymer Nanocomposite

- Energy Filtering Effect

2. Experimental Work

- $\mathrm{Mn}_{3} \mathrm{O}_{4}$ Nanoparticles

- Nanoparticle Functionalization

- Hybrid Film Making

3. Results

- Thermoelectrical Characterization

- Nanoparticle Influence on Polymer Morphology

- Humidity Effect

4. Conclusion and Further Developments 


\section{Thermoelectric}

\section{Results}

\section{Characterization}

\begin{tabular}{|c|c|c|c|}
\hline & $\begin{array}{c}{[\mathrm{NP}]} \\
\left(\mathrm{cm}^{-3}\right)\end{array}$ & $\begin{array}{c}\sigma_{\mathrm{dry}} \\
\left(\Omega^{-1} \mathrm{~cm}^{-1}\right)\end{array}$ & $\begin{array}{c}\alpha_{\mathrm{dry}} \\
\left(\mu \mathrm{VK}^{-1}\right)\end{array}$ \\
\hline PEDOT:Tos & 0 & $242 \pm 9$ & $15.8 \pm 0.9$ \\
\hline HF1 & $1.5 \cdot 10^{14}$ & $240 \pm 9$ & $14.8 \pm 0.5$ \\
\hline HF2 & $3.0 \cdot 10^{14}$ & $147 \pm 6$ & $15.5 \pm 0.7$ \\
\hline HF3 & $2.2 \cdot 10^{15}$ & $98 \pm 4$ & $15.0 \pm 0.2$ \\
\hline HF4 & $3.0 \cdot 10^{15}$ & $89 \pm 3$ & $15.5 \pm 0.8$ \\
\hline & \multicolumn{3}{|c|}{$\sigma=e p \mu$} \\
\hline
\end{tabular}

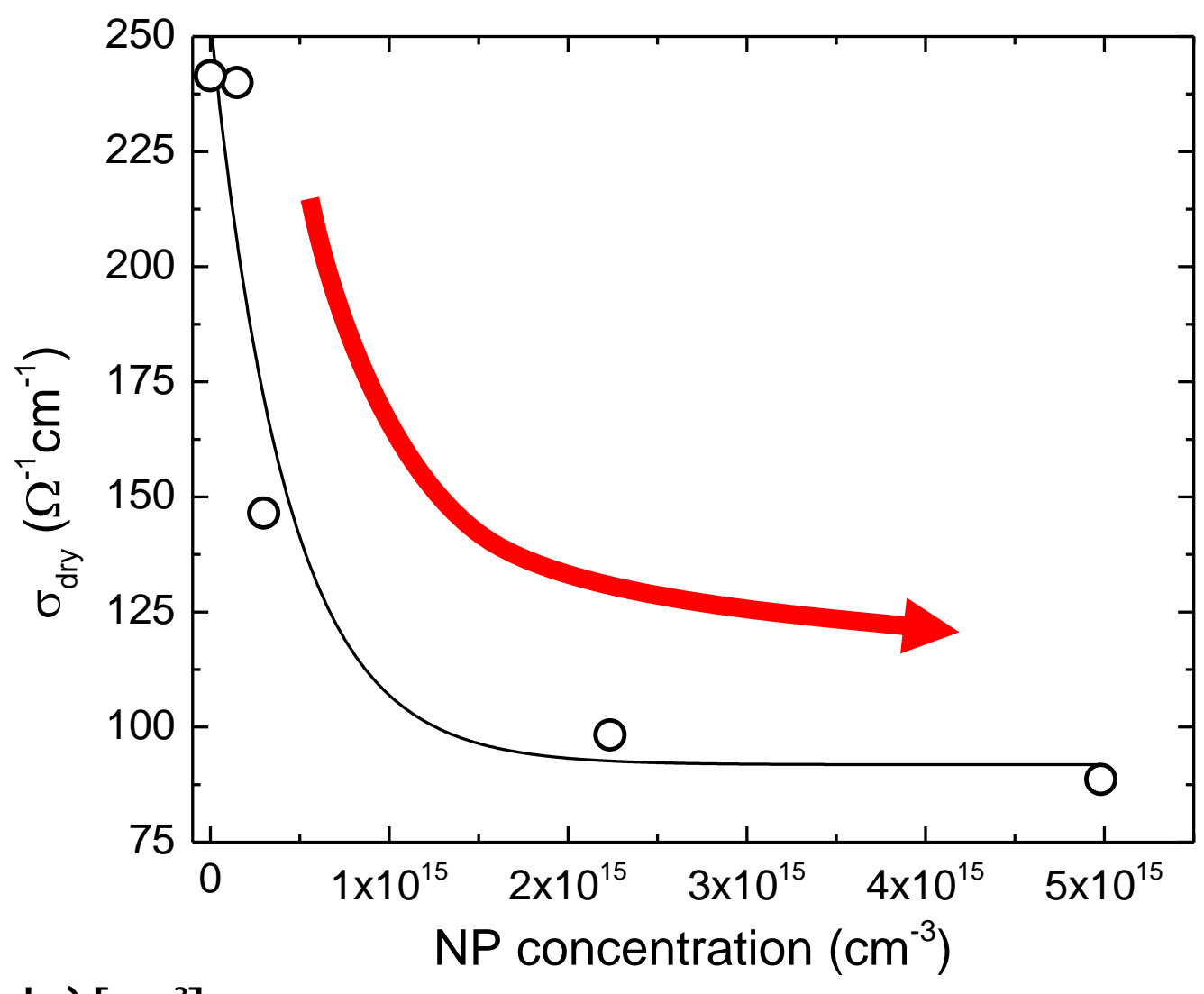

[NP]: NP density

$\sigma$ : electrical conductivity

$\alpha$ : Seebeck coefficient

e: electronic charge

$\left(1.6 \times 10^{-19} \mathrm{C}\right)$ $\mathrm{p}$ : charge carrier density (holes) $\left[\mathrm{cm}^{-3}\right]$ $\mu$ : charge carrier mobility $\left[\mathrm{cm}^{2} /(\mathrm{V} \cdot \mathrm{s})\right]$

$\mu_{0}, \mu_{1}, \mathrm{~N}_{0}$ : constants 


\section{Nanoparticle Influence on Polymmer} Results

\section{Morphology}
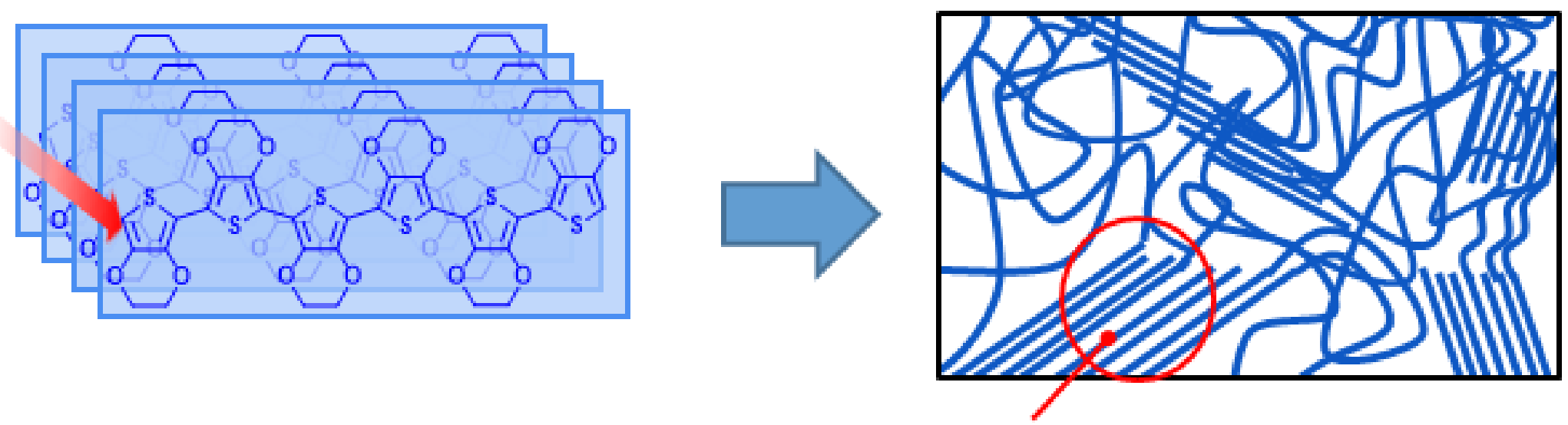

Crystalline domain
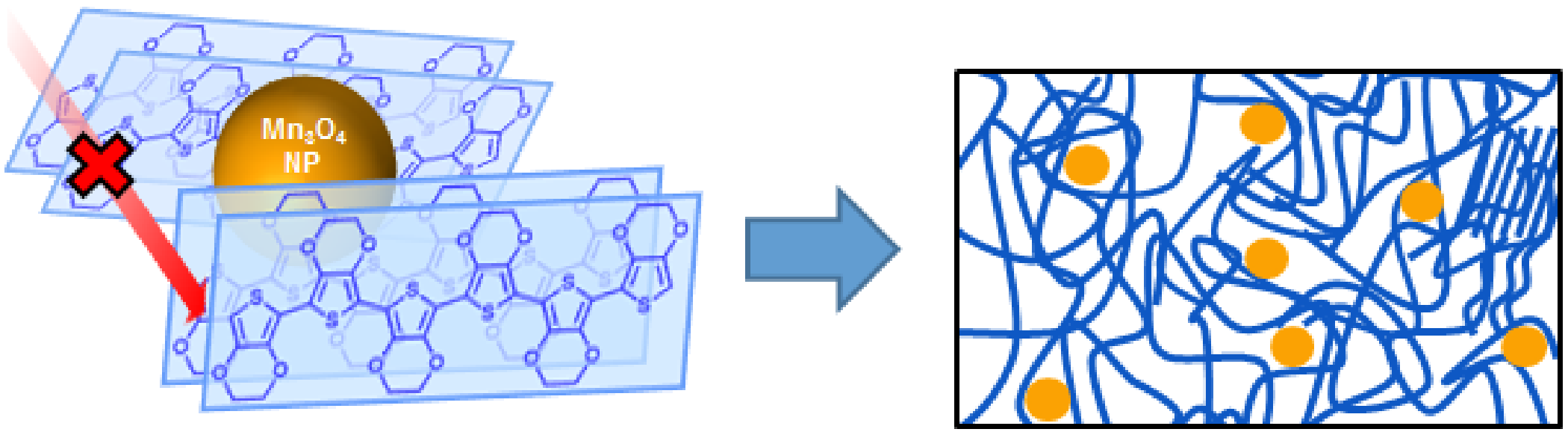


\section{Results}

\section{Detrimental effect on $\sigma$ :}

Water interposition between polymer chains

\section{$0.3-0.4 \mathrm{~nm}$}

Negligjible in

$\mathrm{NP}$

\section{Humidity Effect}

\section{Beneficial effect on $\sigma$ :}

Counterion solvatation

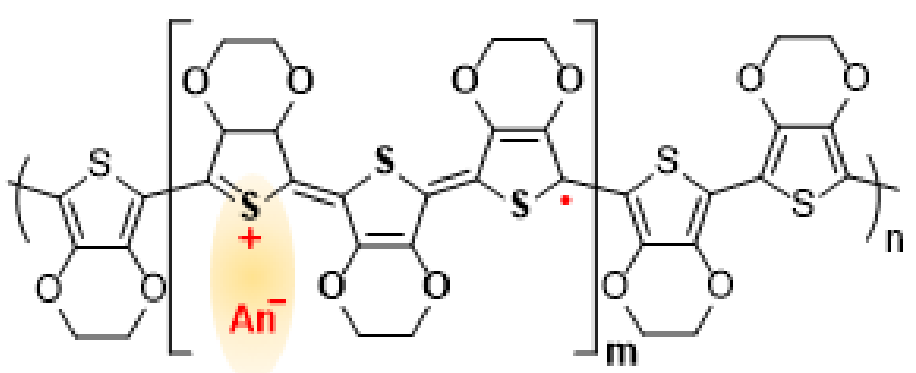




\section{Humidity Effect}

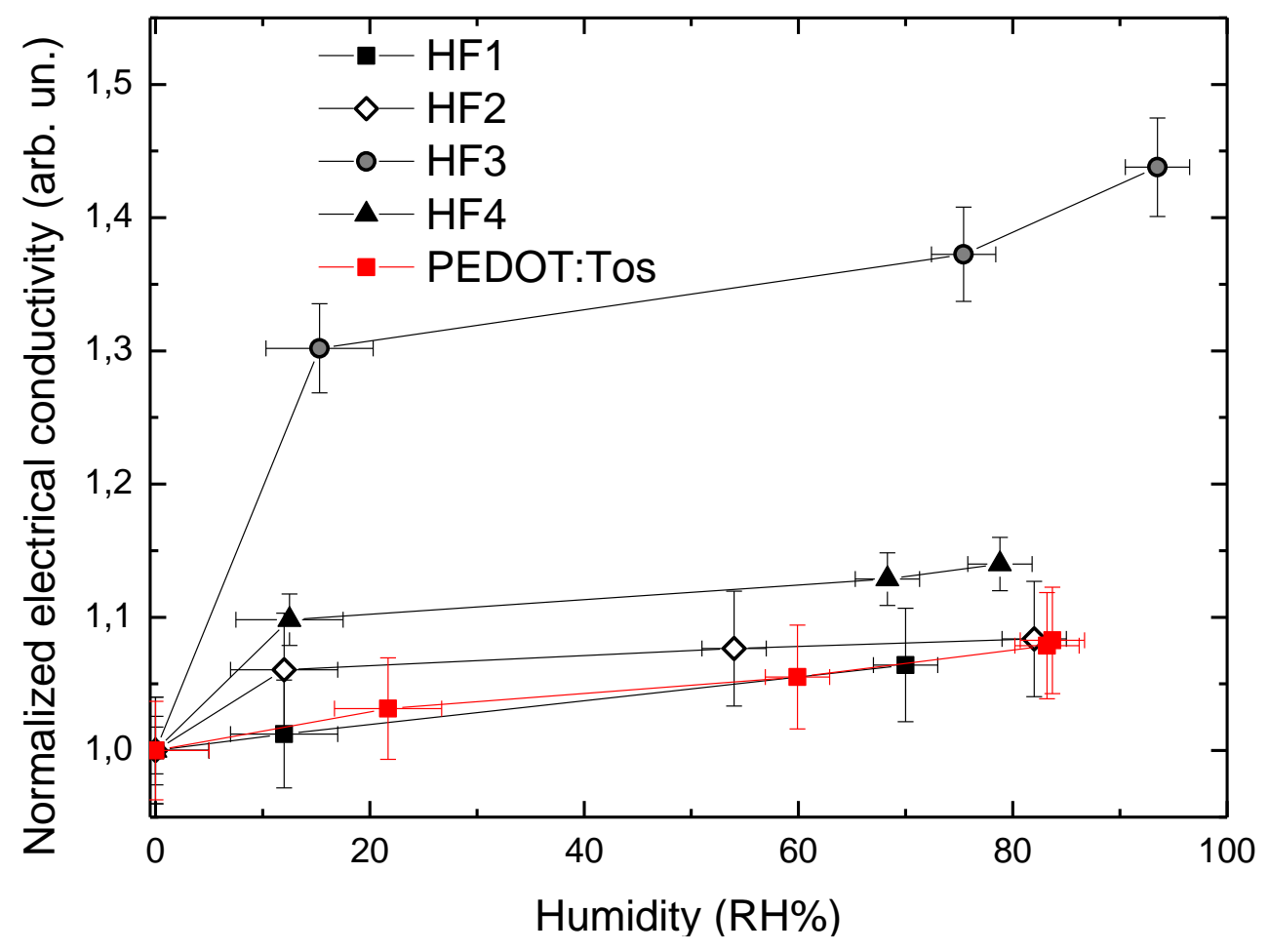

[NP]: NP density $\sigma$ : electrical conductivity e: electronic charge $\left(1.6 \times 10^{-19} \mathrm{C}\right)$ $\mathrm{x}_{\mathrm{w}}$ : water molar fraction $\beta$ : dimensionless function $(\beta>0)$

$$
\sigma-\sigma_{\text {dry }}=\beta([N P]) x_{\mathrm{w}}\left(\sigma_{0}+\sigma_{1} e^{-[N P] / N_{0}}\right)
$$


Results

\section{Humidity Effect}

$$
\sigma-\sigma_{\mathrm{dry}}=\beta \xi\left(\mathrm{RH} ; \delta_{x}, x_{0}\right)\left(\sigma_{0}+\sigma_{1} e^{-[N P] / N_{0}}\right)
$$

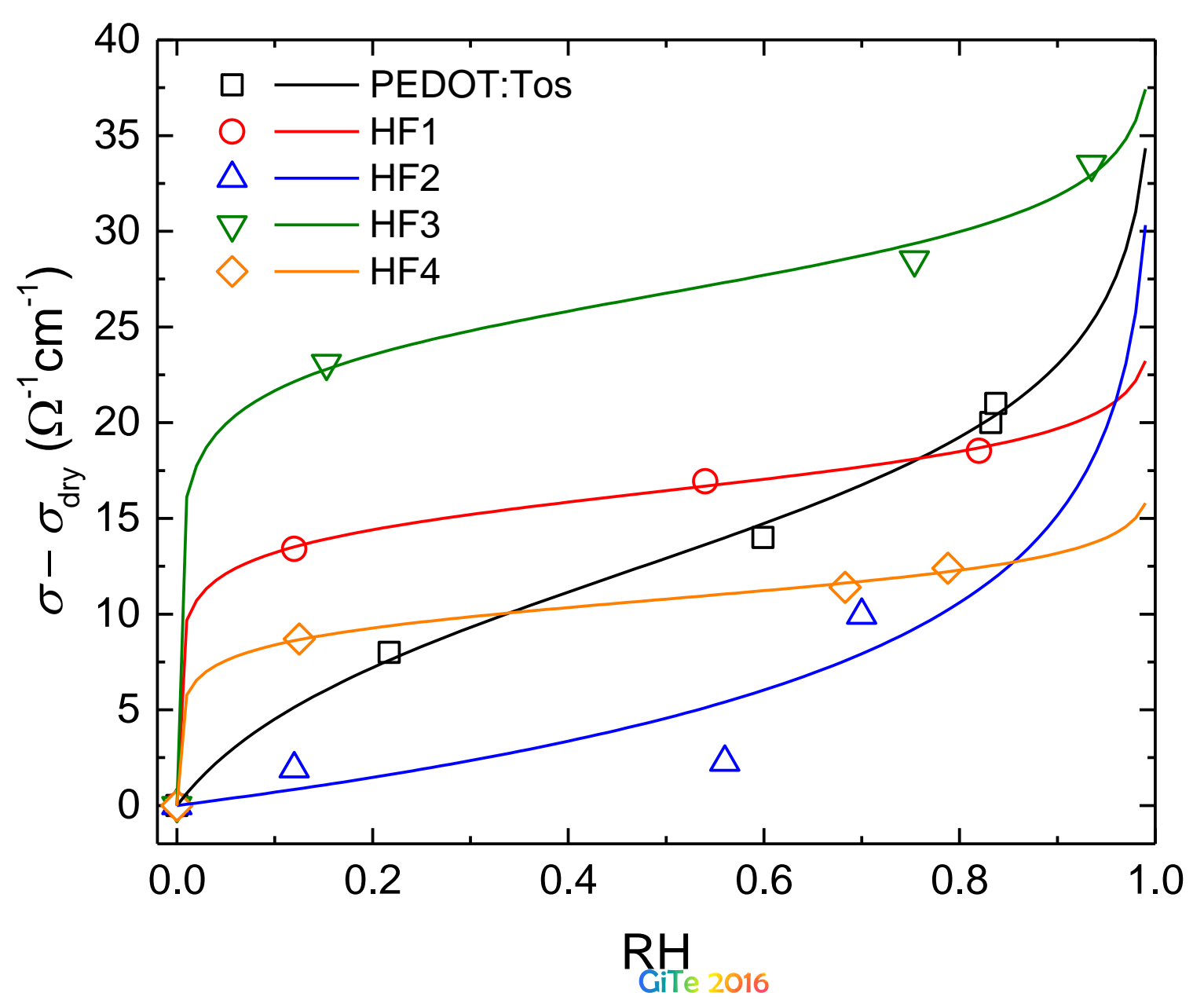

[NP]: NP density $\sigma$ : electrical conductivity $\beta, \delta_{x}, x_{0}$ : model parameters $\mathrm{RH}$ : relative humidity 


\section{Humidity Effect}

Results

\section{Understanding parameters}

$$
\sigma-\sigma_{\text {dry }}=\beta \xi\left(\mathrm{RH} ; \delta_{x}, x_{0}\right)\left(\sigma_{0}+\sigma_{1} e^{-[N P] / N_{0}}\right)
$$

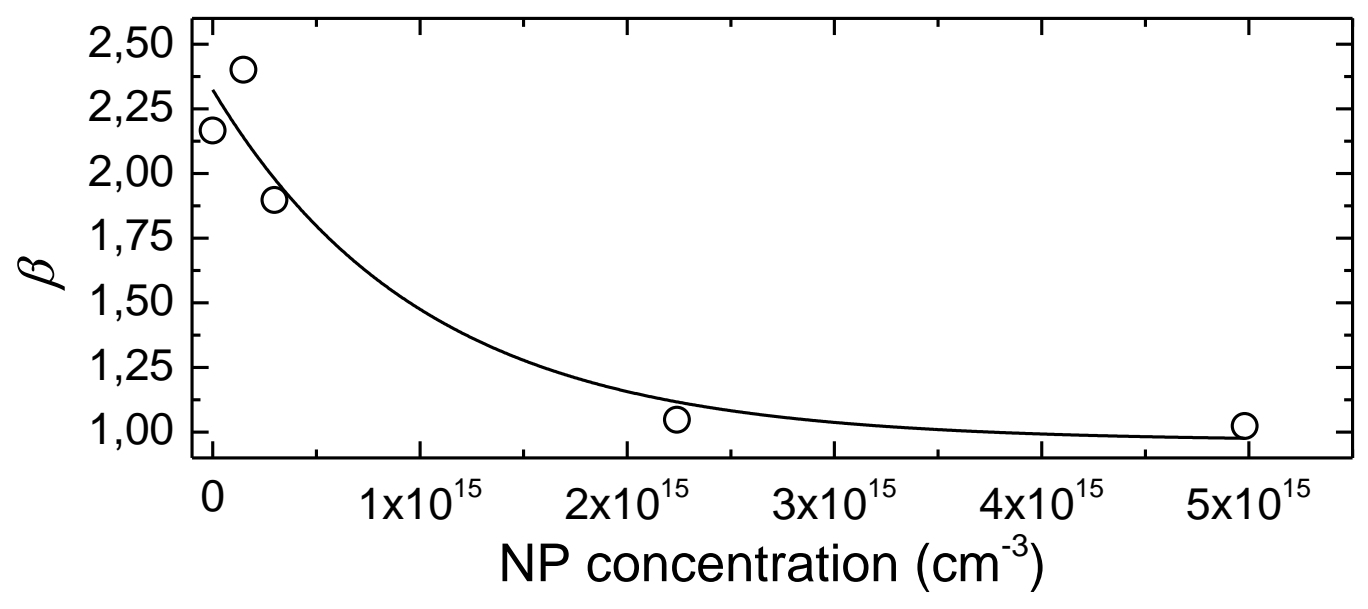

Parameter $\boldsymbol{\beta}$ (variation of $p$ due to $x_{w}$ ) exponential decay us [NP]

\section{Water molecules sequestration} by NPs 


\section{Conclusion}

\section{Conclusions and Further}

\section{Developments}

Results obtained:

- A novel protocol to obtain hybrid material CP/INPs has been developed

- Understanding of morphology related aspects of the developed system

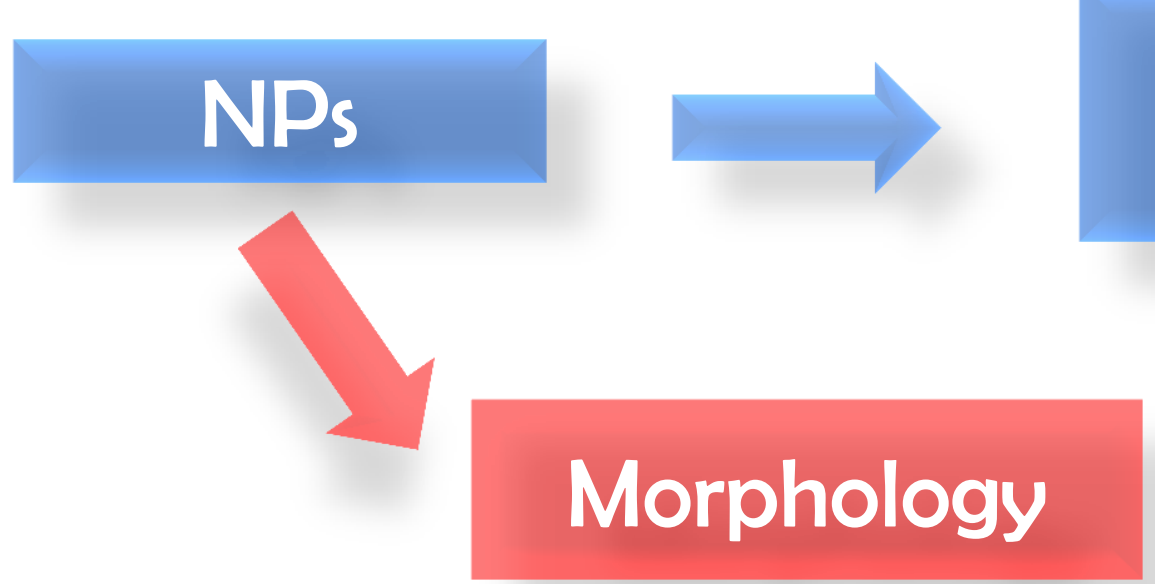

\section{Electrical \\ properties}




\section{Conclusion}

\section{Conclusions and Further Developments}

Further Developments:

- Development of a strategy to avoid nanomaterial detrimental effect on morphology

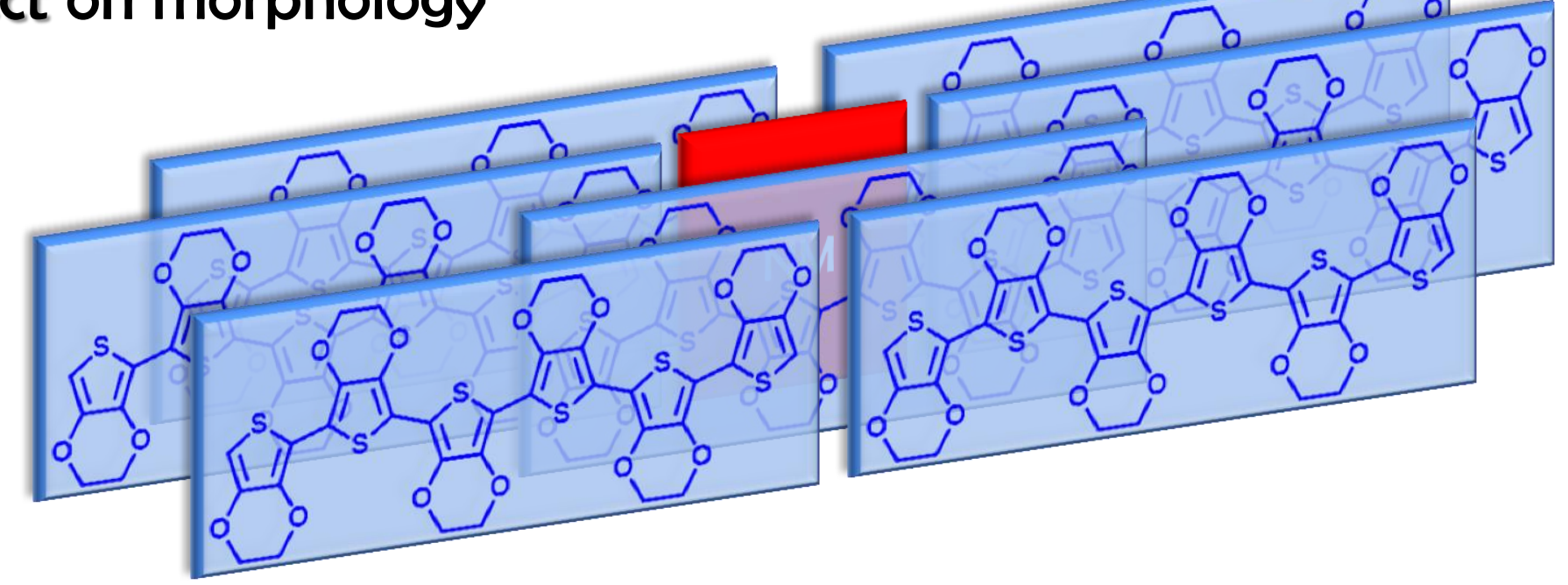

1. Implement polymerization and post-polymerization treatments to favor the rearrangements of NPs (head-to-tail)

2. Using 1D nanomaterial 


\section{Thank you for your kind attention!}

\section{Aknowledgments}

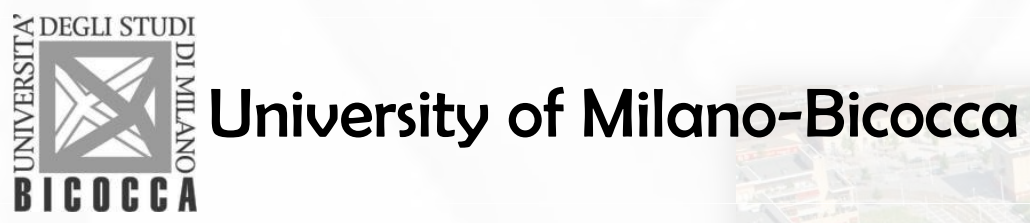

Thermoelectrics Group:

Professor Dario Narducci, Dr. Bruno Lorenzi, Dr. Laura Zulian

LaSMO Group (Organic Synthesis):

Professor Luca Beverina, Dr. Mauro Sassi

Electrochemistry Group:

Professor Riccardo Ruffo

AFM Characterization:

Dr. Silvia Trabattoni

Co-Supervisor:

Dr. Luca Bertini
IENI-CNR Padova

SEM Characterization:

Dr. Simone Battiston

University of Pavia

Co-Supervisor: Professor Umberto Anselmi-Tamburini 


\section{Further explanations \\ Conductivity in CP}

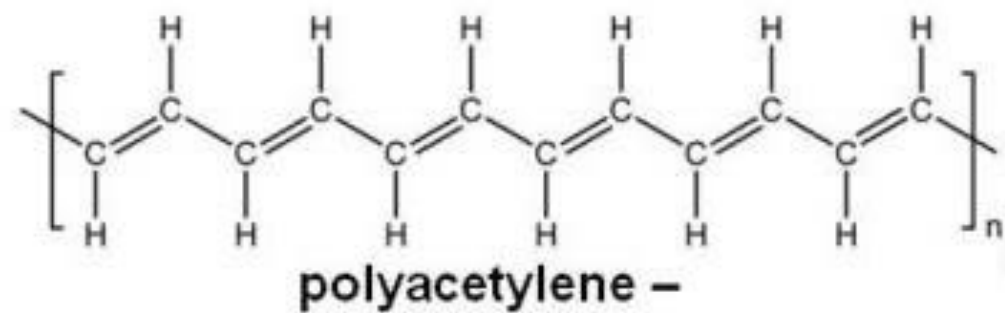

an approximately linear structure

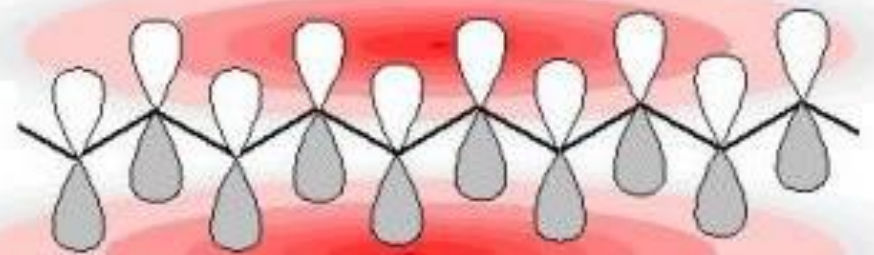

$p_{z}$ orbitals in polyacetylene

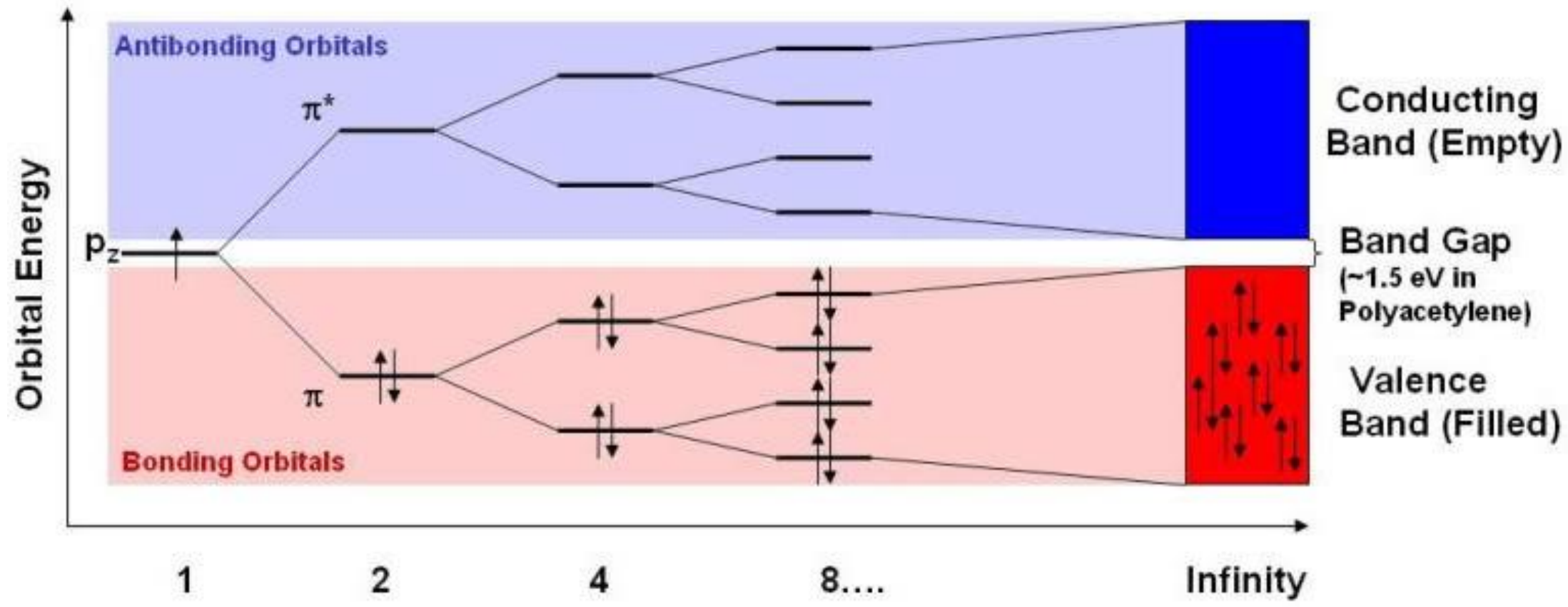




\section{Further explanations \\ NP IR Characterization}

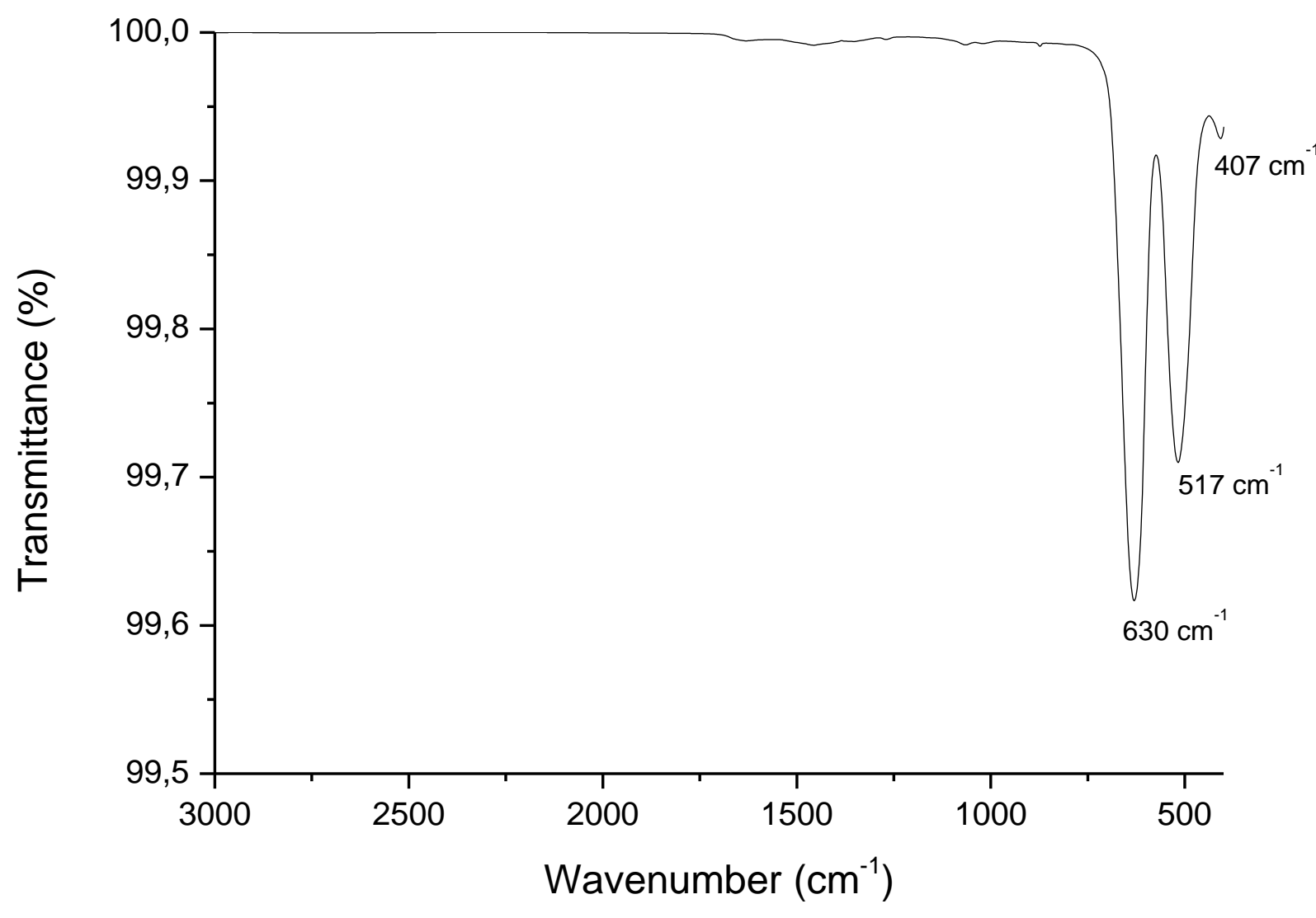

- $630 \mathrm{~cm}^{-1}$

Mn-O stretching modes in tetrahedral sites

- $517 \mathrm{~cm}^{-1}$

$\mathrm{Mn}-\mathrm{O}$ stretching modes in octaedral sites

- $407 \mathrm{~cm}^{-1}$

$\mathrm{Mn}^{3+}-\mathrm{O}$ vibrational modes in octahedral sites 
Further explanations

\section{Synthesis of glutaryl-EDOT}

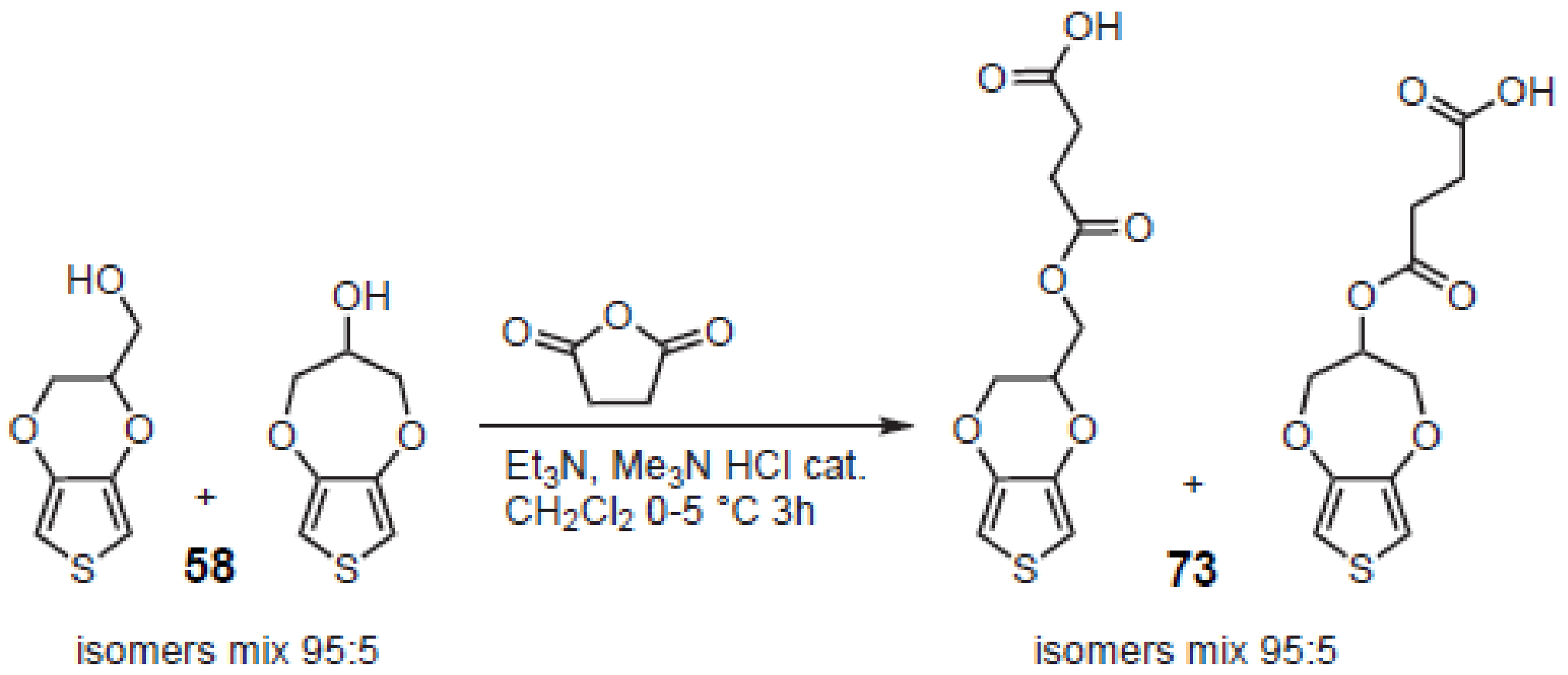




\section{Further explanations \\ UV-vis Characterization of decorated NP}
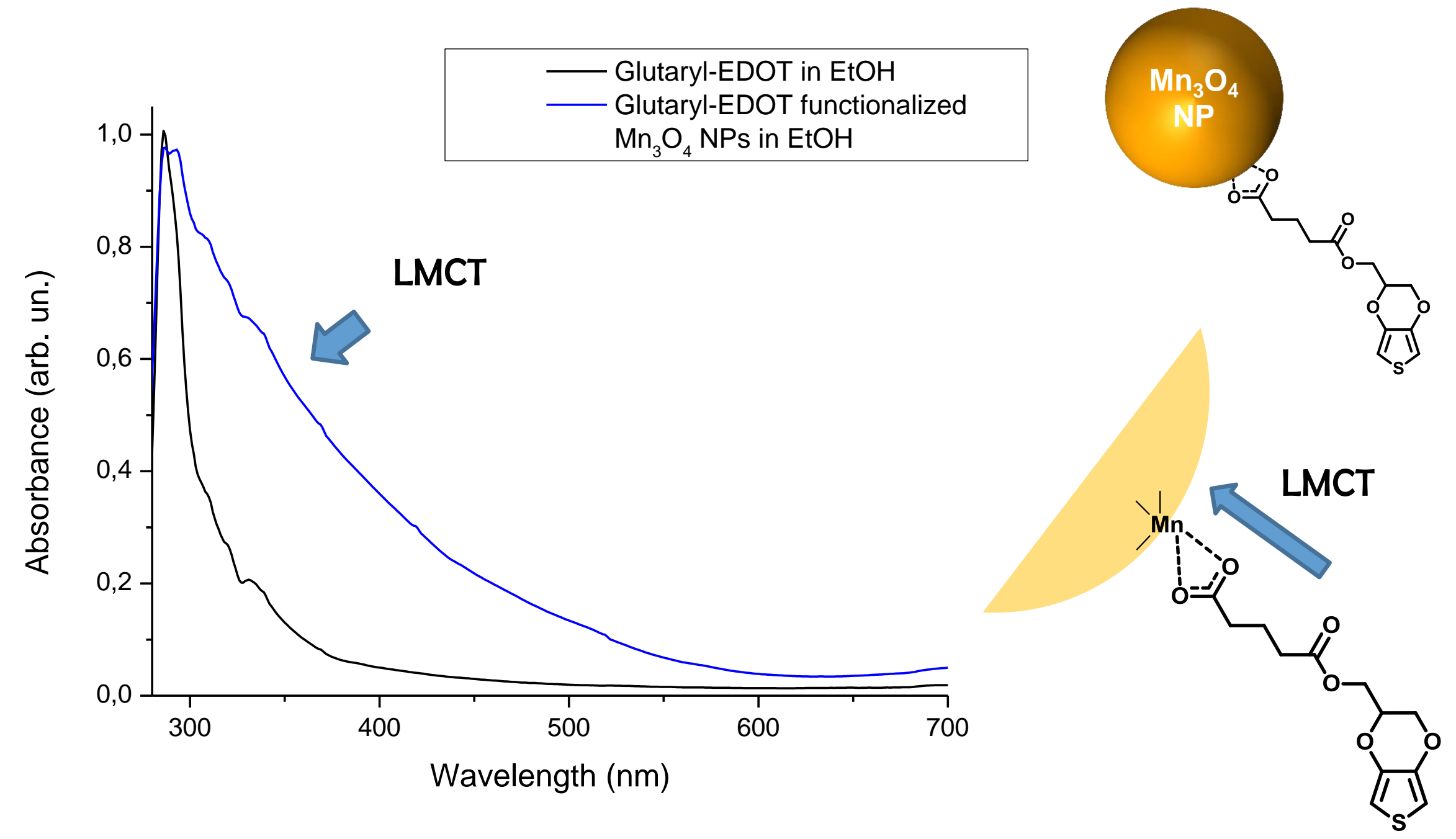
Further explanations

\section{Power Factor Values}

\begin{tabular}{ccccc}
\hline PEDOT:Tos & 0 & $242 \pm 9$ & $15.8 \pm 0.9$ & $6.0 \pm 0.8$ \\
HF1 & $1.5 \cdot 10^{14}$ & $240 \pm 9$ & $14.8 \pm 0.5$ & $5.5 \pm 0.7$ \\
HF2 & $3.0 \cdot 10^{14}$ & $147 \pm 6$ & $15.5 \pm 0.7$ & $3.8 \pm 0.5$ \\
HF3 & $2.2 \cdot 10^{15}$ & $98 \pm 4$ & $15.0 \pm 0.2$ & $2.2 \pm 0.6$ \\
\hline HF4 & $3.0 \cdot 10^{15}$ & $89 \pm 3$ & $15.5 \pm 0.8$ & $2.0 \pm 0.3$ \\
\hline
\end{tabular}




\section{Humidity Effect}

\section{Further} explanations

\section{Understanding the model}

$$
\begin{gathered}
\sigma=e p\left(x_{\mathrm{w}},[N P]\right)\left(\mu_{0}+\mu_{1} e^{-[N P] / N_{0}}\right) \quad \Rightarrow p\left(x_{\mathrm{w}},[N P]\right)=p(0,[N P])+\left(\frac{\partial p}{\partial x_{w}}\right)_{x_{\mathrm{w}}=0} x_{\mathrm{w}} \equiv p_{0}\left(1+\beta([N P]) x_{\mathrm{w}}\right) \\
\sigma-\sigma_{\text {dry }}=\beta([N P]) x_{\mathrm{w}}\left(\sigma_{0}+\sigma_{1} e^{-[N P] / N_{0}}\right)
\end{gathered}
$$

Rault's equation for non-ideal solution: $\quad P_{\mathrm{w}}^{(0)} / P^{0}=\frac{P_{\mathrm{w}} / P^{0}}{a_{\mathrm{w}}\left(x_{\mathrm{w}}\right)}$

Water activity approximated as a standard sigmoidal:

$$
a_{w}\left(x_{\mathrm{w}}\right)=\operatorname{csch}\left(\frac{1}{2 \delta_{x}}\right) \sinh \left(\frac{x_{\mathrm{w}}}{2 \delta_{x}}\right) \cosh \left(\frac{x_{0}-1}{2 \delta_{x}}\right) \operatorname{sech}\left(\frac{x_{\mathrm{w}}-x_{0}}{2 \delta_{x}}\right)
$$

Water molar fraction according to the water activity formula:

$$
x_{\mathrm{w}}=2 \delta_{x} \operatorname{coth}^{-1}\left(\frac{(\mathrm{RH}-1) \tanh \left(\frac{x_{0}}{2 \delta_{x}}\right)+\operatorname{coth}\left(\frac{1}{2 \delta_{x}}\right)}{\mathrm{RH}}\right) \equiv \xi\left(\mathrm{RH} ; \delta_{x}, x_{0}\right)
$$

$$
\sigma-\sigma_{\mathrm{dry}}=\beta \xi\left(\mathrm{RH} ; \delta_{x}, x_{0}\right)\left(\sigma_{0}+\sigma_{1} e^{-[N P] / N_{0}}\right)
$$

\section{[NP]: NP density}

$\sigma$ : electrical conductivity

e: electronic charge $\left(1.6 \times 10^{-19} \mathrm{C}\right)$

$\mathrm{x}_{\mathrm{w}}$ : water molar fraction $\beta$ : dimensionless function $(\beta>0)$

p: charge carrier density (holes) $\left[\mathrm{cm}^{-3}\right]$ $\mu$ : charge carrier mobility $\left[\mathrm{cm}^{2} /(\mathrm{V} \cdot \mathrm{s})\right]$

$\mu_{\mathrm{o}}, \mu_{1}, \mathbf{N}_{\mathrm{o}}$ : constants

$P_{0}: 1$ bar
$\mathbf{P}_{w}{ }^{(0)}$ : equilibrium water pressure

$a_{w}$ : water activity

RH: relative humidity $=\frac{\text { actual vapor density }}{\text { saturation vapor density }} \times 100 \%$ 


\section{Further}

\section{Record ZT value at room temperature}

\section{Engineered doping of organic semiconductors for enhanced thermoelectric efficiency}

\section{G-H. Kim¹, L. Shao', K. Zhang' and K. P. Pipe ${ }^{1,2 \star}$}
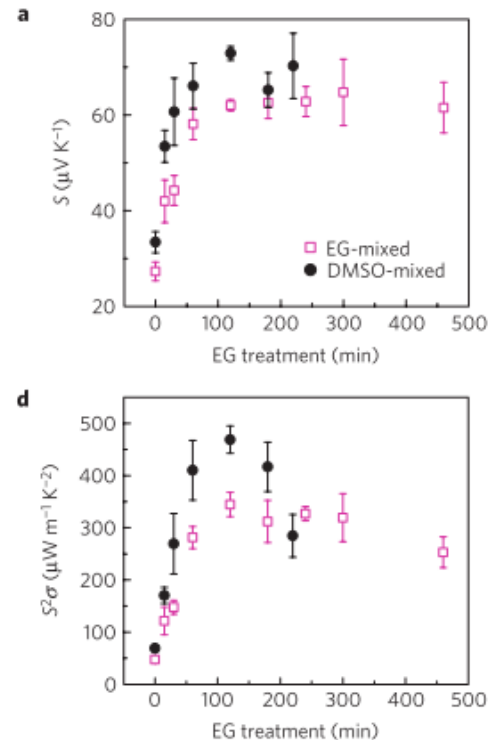

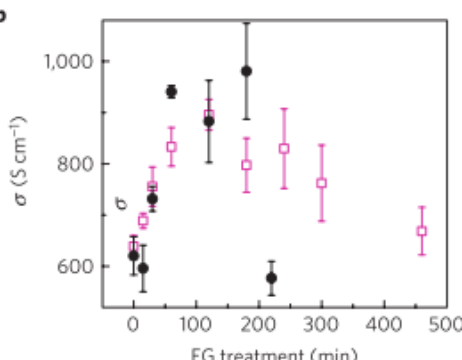

e

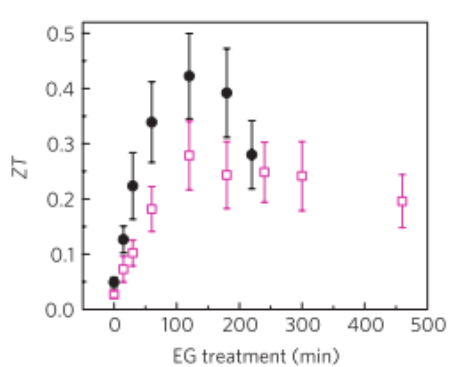

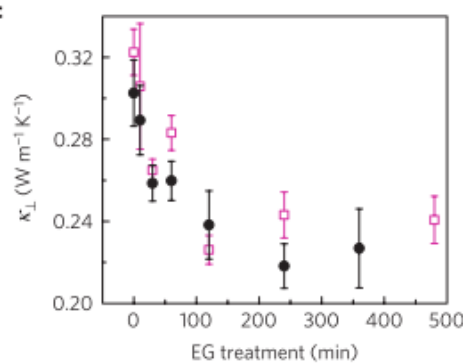

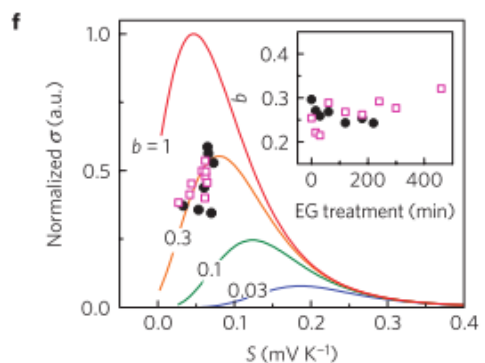

Significant improvements to the thermoelectric figure of merit ZT have emerged in recent years, primarily due to the engineering of material composition and nanostructure in inorganic semiconductors' (ISCs). However, many present high-ZT materials are based on low-abundance elements that pose challenges for scale-up, as they entail high material costs in addition to brittleness and difficulty in large-area deposition. Here we demonstrate a strategy to improve $Z T$ in conductive polymers and other organic semiconductors (OSCs) for which the base elements are earth-abundant. By minimizing total dopant volume, we show that all three parameters constituting $Z T$ vary in a manner so that $Z T$ increases; this stands in sharp contrast to ISCs, for which these parameters have trade-offs. Reducing dopant volume is found to be as important as optimizing carrier concentration when maximizing ZT in OSCs. Implementing this strategy with the dopant poly(styrenesulphonate) in poly(3,4-ethylenedioxythiophene), we achieve $Z T=0.42$ at room temperature. 